\title{
The efficacy of ozone therapy in neonatal rats with hypoxic ischemic brain injury
}

\author{
Resitoglu B ${ }^{1}$, Celik $Y^{2}$, Komur $\mathrm{M}^{3}$, Polat $\mathrm{A}^{4}$, Erdogan $\mathrm{S}^{5}$, Arslankoylu AE ${ }^{6}$, Beydagi $\mathrm{H}^{7}$ \\ Mersin University School of Medicine, Department of Neonatal Intensive Care Unit, Mersin, Turkey. \\ drycelik@yahoo.com
}

\section{ABSTRACT}

OBJECTIVES: This study is aimed to determine the effect of ozone therapy in neonatal rats with experimentally induced hypoxic ischemic brain injury (HIBI).

METHODS: The study included 7-d-old male Wistar rats that were randomized to the sham, control, ozone 1, and ozone 2 groups. All rats except those in the sham group were kept in a hypoxia chamber, and then the rats in the control group were given $0.5 \mathrm{~mL}$ of saline. Those in the ozone 1 group were given ozone $1 \mathrm{mg} \mathrm{kg}^{-1}$ intraperitoneally, and those in the ozone 2 group were given ozone $2 \mathrm{mg} \mathrm{kg}^{-1}$ intraperitoneally.

RESULTS: There were significantly fewer apoptotic neurons in the right hemispheres of the rats in the ozone 1 and ozone 2 groups than in the control group ( $p<0.001$ and $p<0.001$, respectively). There were significantly fewer apoptotic neurons in the right hemispheres of the rats in the ozone 2 group than in the ozone 1 group ( $p$ $<0.001)$. Morris Water Maze (MWM) test results were similar in the ozone 2 and sham groups.

CONCLUSIONS: The present study's findings show that ozone therapy reduced neuronal apoptosis and improved cognitive function in neonatal rats with experimentally induced HIBI (Tab. 2, Ref. 30). Text in PDF www.elis.sk. KEY WORDS: hypoxic ischemic brain injury, neuroprotection, ozone.

\section{Introduction}

Moderate to severe hypoxic ischemic encephalopathy (HIE) occurs in approximately 1-3/1000 live births, and is a major cause of neonatal death and disability (1). Recent multicenter randomized controlled studies have shown that therapeutic hypothermia (TH) decreases mortality and disability in infants with HIE (2); however, TH is not always sufficient, especially in cases of severe HIE, as very high number of infants die or suffer from disability despite receiving $\mathrm{TH}$ (3). As such, more efficient treatment methods that can be used with or without TH are needed for patients with HIE.

Ozone $\left(\mathrm{O}_{3}\right)$ is a cyclic gas composed of 3 oxygen atoms (4). Recently, medical ozone therapy has been shown to be a safe, economic, and effective treatment for many conditions, including advanced ischemic lesions, peritonitis, infected wounds, chronic skin ulcers, initial gangrene, and burns (5). Some animal studies reported that ozone inhibited the apoptosis and exhibited an antiinflammatory effect in ischemia-reperfusion (I/R) injury experi-

${ }^{1}$ Mersin University Vocational School of Health Services, Mersin, Turkey, ${ }^{2}$ Mersin University School of Medicine, Department of Neonatal Intensive Care Unit, Mersin, Turkey, ${ }^{3}$ Mersin University School of Medicine, Department of Pediatric Neurology, Mersin, Turkey, ${ }^{4}$ Mersin University School of Medicine, Department of Pathology, Mersin, Turkey, ${ }^{5}$ Mersin University Faculty of Medicine, Department of Biostatistics, Mersin, Turkey, ${ }^{6}$ Mersin University School of Medicine, Department of Pediatrics, Mersin, Turkey, and ${ }^{7}$ Mersin University School of Medicine, Department of Physiology, Mersin, Turkey

Address for correspondence: Y. Celik, Mersin University School of Medicine, Department of Neonatal Intensive Care Unit, 33343, Mersin, Turkey. Phone: +90.324.2410000-2618, Fax: +90.324.2410098 mentally induced in several organs $(5,6)$. In addition, studies have shown that ozone reinforced the endogenous antioxidant systems and protected organ functions by ensuring adaptation against transient oxidative stress $(5,7)$. A recent in vitro rat study reported that ozone exerted a neuroprotective effect in a brain ischemia model (8, 9 ). It was suggested that ozone exerts its neuroprotective activity via a kind of preconditioning effect by initiating repair on one hand and increasing the defensive capacity through a hormesis effect on the other (9). To the best of our knowledge, literature does not include any studies on the effect of ozone in a neonatal hypoxic ischemic brain injury (HIBI) model; therefore, the present study is aimed to determine the effect of ozone therapy in neonatal rats with experimentally induced HIBI.

\section{Material and methods}

\section{Experimental procedures}

The study was conducted by the Pediatrics, Physiology, and Pathology Departments of Mersin University School of Medicine, at the Experimental Animals Research Laboratory and Physiology Behavior Laboratory. The Mersin University Animal Experiments Ethics Committee approved the study protocol. 7-d-old male Wistar rats $(n=64)$ were used in the present study. They were randomized into 4 groups:

Sham group $(\mathrm{n}=16)$ : Following neck dissection, the right carotid artery was located, but not tied. Hypoxia was not induced;

Control group $(n=16)$ : Immediately following induction of HIBI, $0.4 \mathrm{~mL}$ of saline was administered via the intraperitoneal route; 
Ozone 1 group $(n=16)$ : Immediately following induction of HIBI, ozone $1 \mathrm{mg} \mathrm{kg}^{-1}$ was administered via the intraperitoneal route;

Ozone 2 group $(\mathrm{n}=16)$ : Immediately following induction of HIBI, ozone $2 \mathrm{mg} \mathrm{kg}^{-1}$ was administered via the intraperitoneal route.

All rats were administered inhalation anesthesia for $<5 \mathrm{~min}$, and then a midline neck incision was performed and the right carotid artery was located via microscopic guidance. In all rats, except those in the sham group, the right carotid artery was tied with 6.0 silk suture and the rats were placed in a hypoxia chamber containing $8 \%$ oxygen for $2 \mathrm{~h}$. Next, the rats were removed from the chamber and administered treatment according to group, and then placed with their mothers for a 2-h recovery period (10).

Following $2 \mathrm{~h}$ of recovery, 8 rats from each group were euthanized via cervical dislocation and were decapitated. In order to observe neuronal apoptosis, the rats' brains were removed, and then examined via the terminal deoxynucleotidyl transferase-mediated deoxyuridine triphosphate nick-end labeling (TUNEL) method. The remaining rats underwent Morris water maze (MWM) testing at age 14 weeks.

\section{Ozone generation and administration}

Evozone basic Plus (Reutlingen, Germany) was used for ozone generation (flow rate: $10 \mathrm{~mL} \mathrm{~s}^{-1}$; concentration range: $0-80 \mu \mathrm{g}$ $\mathrm{mL}^{-1}$ ). Ozone at the concentration of $25 \mu \mathrm{g} \mathrm{mL}^{-1}$ was used for the study. Ozone was collected from the generator in ozone-resistant injectors and was immediately administered to the rats intraperitoneally at $1 \mathrm{mg} \mathrm{kg}^{-1}$ or $2 \mathrm{mg} \mathrm{kg}^{-1}$.

\section{Evaluation of apoptosis}

In order to evaluate apoptosis, two samples from the parietal cortex, hippocampus, and subthalamic nucleus were obtained from each rat. A pathologist that was blinded to the groups and to which carotid artery was tied examined the rat brains. DNA fragmentation in neurons was observed by using the TUNEL method (in situ apoptosis detection kit, Biogen, catalog no. S7101). Firstly, deparaffinized coronal brain sections ( $5 \mathrm{~mm}$ thick) were treated with alcohol, after that they were incubated with protein kinase $\mathrm{K}$ at room temperature for $15 \mathrm{~min}$. Next, endogen peroxidase activity was extinguished using $2 \% \mathrm{H}_{2} \mathrm{O}_{2}$, and then slices were incubated at $37{ }^{\circ} \mathrm{C}$ for $60 \mathrm{~min}$ in a moist chamber with $50 \mu \mathrm{L}$ of TdT buffer. Following incubation, the reaction was made visible using a streptavidin-biotin-peroxidase complex and diaminobenzidine. 1 $\%$ methyl green was used to counter stain TUNEL-labeled slides. In order to evaluate numeric density, total TUNEL-positive neurons were enumerated in 5 high-power fields $\left(400^{\prime}\right)$ via light microscopy. Samples from the subthalamic nucleus, hippocampus, and parietal cortex of both the right and left hemispheres were used to enumerate apoptotic neurons (11).

\section{MWM test}

The rats were placed in the behavioral experiments laboratory 2 days prior to MVM testing for habituation. MWM testing was initiated at age 14 weeks and was performed for 5 days. Water $\left(22{ }^{\circ} \mathrm{C}\right)$ was used to fill a $42-\mathrm{cm}$ deep tank. The same investigator placed the rats in the tank during each MVM test. All rats underwent MWM testing between 0900 and 1400. The tank's image was transferred to a screen of a computer and this image was divided into 4 equal quadrants: north, south, east, and west.

In the first 4 days of testing, a $15-\mathrm{cm}$ diameter platform was placed in the middle of the east quadrant at a height of $40 \mathrm{~cm}$, so it could not be observed from outside of the 42-cm deep tank. On day 1 of testing, all rats were placed in each quadrant (beginning in the west quadrant and proceeding clockwise) with their heads turned toward the wall of the water maze. On days $2-4$, the rats were placed first in the north, east and south quadrants first, respectively, proceeding in clockwise fashion to the other quadrants, as on day 1.

After being dropped into the water the rats were expected to find the hidden platform within $60 \mathrm{~s}$. Rats that did not locate the platform within $60 \mathrm{~s}$ were guided by hand to the platform and were held there for $15 \mathrm{~s}$. For each d/quadrant platform finding time (PFT) of the rats was recorded. On the $5^{\text {th }}$ day of the experiment, the hidden platform in the east quadrant was removed. After that, all rats were dropped into the water in the west quadrant and stayed in the water for $60 \mathrm{~s}$. Time (s) that the rats spent in the east quadrant (which previously contained the platform) was recorded (12).

\section{Statistical analysis}

Statistical analysis was performed using SPSS v.11.5 for Windows. The normality of the distribution of continuous data was determined using the Shapiro-Wilk test. Variance analysis was used to identify differences between measurements on days 1 , 2,3 , and 4 in each group, as well as differences between groups according to days. When variance was homogenous one-way ANOVA was used and the Welch test was used when it was not homogenous. Homogeneity of the variances was determined via the Levene test. Tukey's and Games-Howell tests were used for paired comparisons. In addition, inter-group differences in time spent in the east quadrant on day 5 were analyzed using one-way ANOVA and the Welch test. Differences between left and right hemisphere apoptotic neuron counts in each group were determined using the paired samples t test. Inter-group differences between right and left hemisphere measurements were determined using one-way ANOVA and the Welch test. Descriptive statistics are shown as mean $\pm \mathrm{SD}$. P values of $<0.05$ were accepted as statistically significant.

\section{Results}

Two of the 64 rats included in the study died while inducing HIBI. Among the remaining 62 rats, 32 were decapitated for neuronal apoptosis assessment and MWM testing was performed with the 30 rats that remained at age 14 weeks (sham group: $\mathrm{n}=8$; control group: $\mathrm{n}=8$; ozone 1 group: $\mathrm{n}=7$; ozone 2 group: $\mathrm{n}=7$ ).

\section{Neuronal apoptosis}

Except the sham group, right hemispheres were containing significantly more apoptotic neurons than left hemispheres in all 
Tab. 1. The number of TUNEL-positive apoptotic neurons according to group.

\begin{tabular}{lccc}
\hline Group & Right hemisphere & Left hemisphere & $\mathrm{P}_{0}$ \\
\hline Sham & $1.60 \pm 0.84$ & $1.50 \pm 0.85$ & 0.823 \\
Control & $10.40 \pm 2.07$ & $3.90 \pm 1.20$ & $<\mathbf{0 . 0 0 1}$ \\
Ozone1 & $5.70 \pm 0.95$ & $2.40 \pm 1.43$ & $<\mathbf{0 . 0 0 1}$ \\
Ozone2 & $3.40 \pm 0.52$ & $1.80 \pm 0.79$ & $<\mathbf{0 . 0 0 1}$ \\
$\mathrm{P}_{1}$ & $<0.001$ & $<0.001$ & \\
$\mathrm{P}_{2}$ & $<0.001$ & 0.451 & \\
$\mathrm{P}_{3}$ & $<0.001$ & 1.000 & \\
$\mathrm{P}_{4}$ & $<0.001$ & 0.025 & \\
$\mathrm{P}_{5}$ & $<0.001$ & 0.001 & \\
$\mathrm{P}_{6}$ & $<0.001$ & 1.000 & \\
\hline
\end{tabular}

Data given are mean $\pm \mathrm{SD}, \mathrm{P}_{0}$ : Comparison of the number of apoptotic neurons in the left and right hemispheres, $\mathrm{P}_{1}$ : Comparison of the number of apoptotic neurons in the sham and control groups, $\mathrm{P}_{2}$ : Comparison of the number of apoptotic neurons in the sham and ozone 1 groups, $\mathrm{P}_{3}$ : Comparison of the number of apoptotic neurons in the sham and ozone 2 groups, $\mathrm{P}_{4}$ : Comparison of the number of apoptotic neurons in the control and ozone1 groups, $\mathrm{P}_{5}$ : Comparison of the number of apoptotic neurons in the control and ozone 2 groups, $\mathrm{P}_{6}$ : Comparison of the number of apoptotic neurons in the ozone 1 and ozone 2 groups

Tab. 2. Mean MWM PFT on days 1-4.

\begin{tabular}{lccccc}
\hline Groups & $\mathrm{d} 1(\mathrm{~s})$ & $\mathrm{d} 2(\mathrm{~s})$ & $\mathrm{d} 3(\mathrm{~s})$ & $\mathrm{d} 4(\mathrm{~s})$ & $\mathrm{P}_{0}$ \\
\hline Sham & $41.39 \pm 12.08$ & $19.34 \pm 11.76$ & $9.64 \pm 5.87$ & $11.03 \pm 7.51$ & 0.001 \\
Control & $51.85 \pm 10.13$ & $41.78 \pm 18.75$ & $41.59 \pm 25.48$ & $34.68 \pm 23.66$ & 0.260 \\
Ozone1 & $47.54 \pm 11.84$ & $41.89 \pm 15.28$ & $31.61 \pm 18.75$ & $28.51 \pm 17.48$ & 0.152 \\
Ozone2 & $45.29 \pm 7.49$ & $30.88 \pm 9.13$ & $27.22 \pm 15.39$ & $15.33 \pm 9.31$ & $<0.001$ \\
$\mathrm{P}_{1}$ & 0.313 & 0.014 & 0.037 & 0.042 & \\
$\mathrm{P}_{2}$ & 1.000 & 0.010 & 0.033 & 0.077 & \\
$\mathrm{P}_{3}$ & 1.000 & 0.353 & 0.048 & 0.731 & \\
$\mathrm{P}_{4}$ & 1.000 & 1.000 & 0.800 & 0.929 & \\
$\mathrm{P}_{5}$ & 1.000 & 0.428 & 0.543 & 0.207 & \\
$\mathrm{P}_{6}$ & 1.000 & 0.394 & 0.951 & 0.250 &
\end{tabular}

MWM; Morris water maze, PFT; platform finding time, data given are mean $\pm \mathrm{SD}$, $\mathrm{P}_{0}$ : Indicates the difference in PFT from day 1 to day 4 within each group, $\mathrm{P}_{1}, \mathrm{P}_{2}, \mathrm{P}_{3}$, $\mathrm{P}_{4}, \mathrm{P}_{5}$, and $\mathrm{P}_{6}$ indicates the difference in PFT between the sham and control groups, between the sham and ozone 1 groups, between the sham and ozone 2 groups, between the control and ozone 1 groups, between the control and ozone 2 groups, and between the ozone 1 and ozone 2 groups, respectively

groups (Tab. 1). The numbers of right hemisphere apoptotic neurons in the sham, ozone 1 , and ozone 2 groups were significantly lower than in the control group $(\mathrm{p}<0.001, \mathrm{p}<0.001$, and $\mathrm{p}<$ 0.001 , respectively). There were significantly fewer right hemisphere apoptotic neurons in the sham group than in the ozone 1 and ozone 2 groups ( $p<0.001$ and $p<0.001$, respectively). The number of right hemisphere apoptotic neurons in the ozone 2 group was significantly lower than in the ozone 1 group $(p<0.001)$.

The numbers of left hemisphere apoptotic neurons in the sham, ozone 1, and ozone 2 groups were significantly lower than in the control group $(\mathrm{p}<0.001, \mathrm{p}=0.025$, and $\mathrm{p}=0.001$, respectively). The sham, ozone 1 , and ozone 2 groups did not differ significantly in terms of left hemisphere apoptotic neurons $(p>0.05)$.

\section{MWM}

PFT was significantly shorter in the sham and ozone 2 groups on day 4 than on day $1(\mathrm{p}=0.001$ and $\mathrm{p}<0.001$, respectively), whereas in the control and ozone 1 groups PFT values did not change on day 4 from those on day $1(p=0.152$ and $p=0.260$, respectively) (Tab. 2). On day 5 of MWM testing, the rats in the control group spent $18.45 \pm 8.86 \mathrm{~s}$ in the east quadrant (where on days $1-4$ the hidden platform was located), versus $26.02 \pm 5.91 \mathrm{~s}$ in the sham group, $18.44 \pm 5.39 \mathrm{~s}$ in the ozone 1 group, and 24.18 $\pm 2.88 \mathrm{~s}$ in the ozone 2 group. On day 5 , the rats in the sham group spent significantly more time in the east quadrant than the rats in the control and ozone 1 groups $(p=0.026$ and $p=0.016$, respectively). There were no significant differences in time spent in the east quadrant on day 5 between the sham and ozone 2 groups ( $p$ $=0.538)$.

\section{Discussion}

Perinatal asphyxia occurs due to insufficient levels of oxygen and blood supplied to the tissues and organs of fetuses and neonates. Although the precise mechanism of cellular damage following hypoxia and ischemia is not known, energy deficiency, and excessive increases in excitatory neurotransmitters, free oxygen radicals, and lipid peroxidation lead to the cascade of injury events $(13,14)$. Free oxygen radicals are a serious threat to vital organs and tissues, and nucleic acids and polyunsaturated fatty acids in cell walls $(1,15)$. Polyunsaturated fatty acids are negatively affected to the greatest extent from this oxidative damage, which is referred to as lipid peroxidation.

Glutathione peroxidase, catalase, and superoxide dismutase (SOD) are antioxidant enzymes that protect biological structures against free radical-mediated injury (13); however, neonates have insufficient antioxidant enzyme levels and activity (16). In addition, rich in unsaturated phospholipids, the brain is extremely susceptible to oxidative stress (17). Studies have shown that free oxygen radical levels are significantly elevated in cases of HIE and that antioxidant enzyme activity does not increase sufficiently (13). Once the importance of free radical damage in the pathogenesis of HIE was understood, researchers began to study the use of antioxidant agents for the treatment of HIE. It was reported that melatonin, a potent antioxidant, is useful for treating HIE (18), as was erythropoietin, which is known to have antioxidant effects (19). Moreover, it was suggested that the suppression of free radical activity and lipid peroxidation associated with $\mathrm{TH}$ contributes significantly to its neuroprotective activity (20). Based on these findings, we think that while free radical damage plays an important role in the pathogenesis of HIE, neonates with HIE have insufficient endogenous antioxidant capacity, and that limiting the free radical damage will be beneficial in the treatment of HIE. As such, we also think that ozone, which stimulates production of endogenous antioxidant enzymes, might also be beneficial in the treatment of HIE. In fact, earlier studies reported that ozone stimulated the production of antioxidant enzymes, including glutathione peroxidase, catalase, and SOD (7).

In recent years, clinically successful utilization of ozone therapy for such conditions as disc hernia, diabetic foot, and peripheral artery disease have been reported (21-23). Furthermore, ozone therapy was reported to limit the effects of experimentally induced $\mathrm{I} / \mathrm{R}$ injury in multiple organs $(24,25)$. Xing et al (26) reported that tumor necrosis factor- $\alpha$ (TNF- $\alpha$ ), interleukin- $1 \beta$ (IL-1 $\beta)$, IL- 6 , in- 
tercellular adhesion molecule 1 (ICAM-1), and monocyte chemo attractant protein-1 (MCP-1) mRNA levels were significantly suppressed, and that toll-like receptor 4 (TLR4) and nuclear factor kappa $\mathrm{B}(\mathrm{NF}-\mathrm{kB})$ expression was inhibited in response to ozone oxidative preconditioning (ozoneOP) in rats with experimentally induced renal I/R. Based on these findings, Xing et al (26) suggested that ozone OP exerted a potent anti-inflammatory effect via modulation of TLR4 and NF-kappa.

Wang et al (6) reported a significant decrease in renal fibrosis in response to ozone OP in rats with experimentally induced renal I/R. Chen et al (24) reported that ozone OP inhibited the inflammation and apoptosis in rats with renal I/R injury. Peralta et al (25) observed a decrease in hepatic damage with ozone treatment in an experimental I/R model. Güven et al (27) reported that antioxidant levels increased, TNF- $\alpha$ level decreased, and histopathologically observed intestinal damage decreased following administration of intraperitoneal ozone to rats with experimentally induced necrotizing enterocolitis. Sukhotnik et al (5) reported that ozone therapy administered intraperitoneally in adult rats increased intestinal cell production and decreased apoptosis. One study reported that intraperitoneally administered ozone decreased the TNF- $\alpha$ level and increased the SOD level in a rat model of experimental peritonitis (4). Bakkal et al repeatedly administered low doses of intraperitoneal ozone to rats with experimentally induced radiation lung injury, and reported a decrease in TNF- $\alpha$ and IL-1 $\beta$ levels, an increase in SOD activity, and a reduction in lung damage (28).

Zhang et al (29) reported a significant decrease in wound healing time in response to ozone-oxygen therapy in patients with diabetic foot ulcers. Zhang et al (29) also observed a significant increase in the levels of vascular endothelial growth factor (VEGF), transforming growth factor- $\beta$ (TGF- $\beta$ ), and plateletderived growth factor (PDGF) following ozone-oxygen therapy. Based on these findings, Zhang et al (29) suggested that the positive effects of ozone-oxygen therapy on wound healing might be due to an increase in endogenous growth factors. Shehata et al (30) reported that ozone therapy can correct the biochemical changes in the cerebral cortex of rats that occur due to aging. Recently, Frosini et al (9) reported that ischemic brain injury was reduced in response to ozone therapy in an in vitro rat model. To the best of our knowledge the present study is the first to show that intraperitoneal ozone therapy administered to neonatal rats with experimentally induced HIBI decreased neuronal apoptosis and improved cognitive functions; these positive effects of ozone were more pronounced when administered at $2 \mathrm{mg} \mathrm{kg} \mathrm{kg}^{-1}$ than $1 \mathrm{mg} \mathrm{kg}^{-1}$.

An important limitation of the present study is that it did not determine the mechanism by which ozone exerted its neuroprotective activity in neonatal HIBI; however, earlier studies suggest that ozone stimulates production of antioxidant enzymes, suppresses inflammation, stimulates immune and neuroendocrine system activity, and promotes release of growth factors via preconditioning- and hormesis-like effects $(7,9,21)$, which might have been elucidated in the present study if antioxidant enzyme levels, the levels of several growth factors, or the levels of several inflamma- tory biomarkers had been investigated. In conclusion, the present findings show that ozone therapy had a neuroprotective effect in neonatal rats with experimentally induced HIBI; however, additional research is needed to understand how ozone exerts its neuroprotective effect.

\section{References}

1. Wassink G, Gunn ER, Drury PP, Bennet L, Gunn AJ. The mechanisms and treatment of asphyxial encephalopathy. Front Neurosci 2014; 8: 40 .

2. Jacobs SE, Berg M, Hunt R, Tarnow-Mordi WO, Inder TE, Davis PG. Cooling for newborns with hypoxic ischaemic encephalopathy. Cochrane Database Syst Rev 2013. doi: 10.1002/14651858.

3. Jacobs SE, Morley CJ, Inder Tev et al. Whole-body hypothermia for term and near-term newborns with hypoxic-Ischemic encephalopathy: a randomized controlled trial. Arch Pediatr Adolesc Med 2011; 165: 692-700.

4. Azuma K, Mori T, Kawamoto K et al. Anti-inflammatory effects of ozonated water in an experimental mouse model. Biomed Rep 2014; 2 : $671-674$

5. Sukhotnik I, Starikov A, Coran AG, Pollak Y, Sohotnik R, Shaoul R. Effect of ozone on intestinal epithelial homeostasis in a rat model. Rambam Maimonides Med J 2015; 6:e0006.

6. Wang L, Chen H, Liu XH et al. Ozone oxidative preconditioning inhibits renal fibrosis induced by ischemia and reperfusion injury in rats. Exp Ther Med 2014; 8: 1764-1768.

7. Re L, Mawsouf MN, Menéndez S, León OS, Sánchez GM, Hernández F. Ozone therapy: clinical and basic evidence of its therapeutic potential. Arch Med Res 2008; 39: 17-26.

8. Bocci V, Valacchi G. Nrf2 activation as target to implement therapeutic treatments. Front Chem 2015; 3: 4

9. Zhang S, Jiang W, Ma L, Liu Y, Zhang X, Wang S. Nrf2 Transfection Enhances the Efficacy of Human Amniotic Mesenchymal Stem Cells to Repair Lung Injury Induced by Lipopolysaccharide. J Cell Biochem 2017. doi: $10.1002 /$ jcb. 26322

10. Frosini M, Contartese A, Zanardi I, Travagli V, Bocci V. Selective ozone concentrations may reduce the ischemic damage after a stroke. Free Radic Res 2012; 46: 612-618.

11. Rice JE, Vanucci RC, Brierley JB. The influence of immaturity on hypoxic-ischemic brain damage in the rat. Ann Neurol 1981; 9: 131-141.

12. Zhu C, Wang X, Cheng X, Qiu L, Xu F, Simruner G, Blomgren K. Post-ischemia hypotermia induced tissue protection and diminished apoptosis after neonatal cerebral hypoxia-ischemia. Brain Res 2004; 996: 67-75.

13. Ikeda T, Mishima K, Yoshikawa T, Iwasaki K, Fujiwara M, Xia YX, Ikenoue T. Selective and long-term learning impairment following neonatal hypoxic-ischemic brain insult in rats. Behav Brain Res 2001; 118: $17-25$.

14. Kumar A, Ramakrishna SV, Basu S, Rao GR. Oxidative stress in perinatal asphyxia. Pediatr Neurol 2008; 38: 181-185.

15. Shalak L, Perlman JM. Hypoxic-ischemic brain injury in the term infant-current concepts. Early Hum Dev 2004; 80: 125-141.

16. Safwat MH, El-Sawalhi MM, Mausouf MN, Shaheen AA. Ozone ameliorates age-related oxidative stress changes in rat liver and kidney: effects of pre- and post-ageing administration. Biochemistry 2014; 79: 450-458. 
17. Perrone S, Stazzoni G, Tataranno ML, Buonocore G. New pharmacologic and therapeutic approaches for hypoxic-ischemic encephalopathy in the newborn. J Matern Fetal Neonatal Med 2012; 25: 83-88.

18. Gómez-Crisóstomo NP, Rodríguez Martínez E, Rivas-Arancibia S. Oxidative stres activates the transcription factors FoxO 1a and FoxO $3 \mathrm{a}$ in the hippocampus of rats exposed to low doses of ozone. Oxid Med Cell Longev 2014; 2014: 805764.

19. Shea KL, Palanisamy A. What can you do to protect the newborn brain? Curr Opin Anaesthesiol 2015; 28: 261-266.

20. Wu YW, Gonzalez FF. Erythropoietin: a novel therapy for hypoxic-ischaemic encephalopathy? Dev Med Child Neurol 2015; 57: 34-39.

21. Shankaran S. Hypoxic-ischemic encephalopathy and novel strategies for neuroprotection. Clin Perinatol 2012; 39: 919-929.

22. Bocci V, Zanardi I, Travagli V. Oxygen/ozone as a medical gas mixture. A critical evaluation of the various methods clarifies positive and negative aspects. Med Gas Res 2011; 1: 6 .

23. Andreula C. Ozone therapy. Neuroradiology 2011; 53: 207-209.

24. Chen H, Xing B, Liu X, Zhan B, Zhou J, Zhu H, Chen Z. Ozone oxidativepreconditioning inhibits inflammation and apoptosis in a rat model of renal ischemia/reperfusion injury. Eur J Pharmacol 2008; 581: 306-314.
25. Peralta C, León OS, Xaus C et al. Protective effect of ozone treatment on the injury associated with hepatic ischemia-reperfusion: antioxidantprooxidant balance. Free Radic Res 1999; 31: 191-196.

26. Xing B, Chen H, Wang L, Weng X, Chen Z, Li X. Ozone oxidative preconditioning protects the rat kidney from reperfusion injury via modulation of the TLR4-NF-אB pathway. Acta Cir Bras 2015; 30: 60-66.

27. Guven A, Gundogdu G, Vurucu S, Uysal B, Oztas E, Ozturk H, Korkmaz A. Medical ozone therapy reduces oxidative stress and intestinal damage in an experimental model of necrotizing enterocolitis in neonatal rats. J Pediatr Surg 2009; 44: 1730-1735.

28. Bakkal BH, Gultekin FA, Guven B, Turkcu UO, Bektas S, Can M. Effect of ozone oxidative preconditioning in preventing early radiationinduced lung injury in rats. Braz J Med Biol Res 2013; 46: 789-796.

29. Zhang J, Guan M, Xie C, Luo X, Zhang Q, Xue Y. Increased growth factors play a role in wound healing promoted by noninvasive oxygenozone therapy in diabetic patients with foot ulcers. Oxid Med Cell Longev 2014; 2014: 273475.

30. Shehata NI, Abd-Elgawad HM, Mawsouf MN, Shaheen AA. The potential role ofozone in ameliorating the age-related biochemical changes in male rat cerebral cortex. Biogerontology 2012; 13: 565-581.

Received September 28, 2017. Accepted October 30, 2017. 